\title{
Linking human factors to corporate strategy with cognitive mapping techniques
}

\author{
Judy Village*, Michael Greig, Filippo A. Salustri and W. Patrick Neumann \\ Human Factors Engineering Lab, Department of Mechanical and Industrial Engineering, Ryerson University, \\ 350 Victoria St., Toronto, Ontario M5B 2K3 Canada
}

\begin{abstract}
For human factors (HF) to avoid being considered of "side-car" status, it needs to be positioned within the organization in such a way that it affects business strategies and their implementation. Tools are needed to support this effort. This paper explores the feasibility of applying a technique from operational research called cognitive mapping to link HF to corporate strategy. Using a single case study, a cognitive map is drawn to reveal the complex relationships between human factors and achieving an organization's strategic goals. Analysis of the map for central concepts and reinforcing loops enhances understanding that can lead to discrete initiatives to facilitate integration of HF. It is recommended that this technique be used with senior managers to understand the organizations`strategic goals and enhance understanding of the potential for HF to contribute to the strategic goals.
\end{abstract}

Keywords: Cognitive mapping, Strategic goals, Human Factors

\section{Introduction and Problem Description}

The benefits from the application of human factors (HF) are frequently under-capitalized in organizations. For HF to avoid being considered an extra or as some have suggested having "side-car" status, it should be positioned so as to directly affect business strategies $[4,5]$. While the literature suggests HF should have a strategic role, and that management support for HF is necessary, it is less clear about how to achieve this. Tools and techniques to help senior management link HF to strategic goals are needed. As a means to do this, we suggest borrowing a technique from the operational research and management fields. The purpose of our work is to demonstrate with a single case study, the utility and feasibility of cognitive mapping to reveal the complex relationships between HF and strategic goals in the organization. The technique has multiple potential benefits. It can provide insight for HF practitioners about the strategic goals of the organization and how senior managers align HF with strategic goals. It can also reveal gaps in understanding concerning HF. The outcome of the mapping reveals action items that can lead to discrete initiatives that would facilitate integration of HF into production systems design. This paper will explain the technique and show a portion of a cognitive map from a single case study to illustrate its potential.

\section{Cognitive Mapping Methodology}

Cognitive mapping has been used in management sciences to address complex problems, for example investigating contributing factors to a large overrun on an engineering project, and to facilitate decisionmaking and negotiation $[9,14]$. When compared with other elicitation techniques, such as surveys, structured interviews, systems mapping, comparison matrices, and soft systems methodologies, mapping has been shown to rate especially high for truthfulness and richness of data, amenable to both qualitative and quantitative analysis [2].

${ }^{*}$ Corresponding author. E-mail: jvillage@ryerson.ca 
In this illustrative case study, used to explore the potential utility of cognitive mapping, a one-hour interview was conducted with one co-author of this paper $(\mathrm{PN})$. The question posed during the interview was "how can integrating HF into design processes help a company achieve their strategic goals"? The interviewee, a certified ergonomist, did not represent a company, but responded generically based on experience working with several manufacturing organizations. Mapping was performed using methods suggested by Ackerman et al [1], where concepts (short phrases) are written down on a large paper as the interviewee responds in a hierarchy with strategic goals at the top of the page, main themes in the middle of the page, and action items towards the bottom. Figure 1 portrays this hierarchical alignment of concepts. Concepts are then connected by directional arrows to show the path of interaction and options as a thread progresses between the question and strategic goal. Connections are always causal where one concept leads to another. During the process the interviewee can visually observe the map develop and see his or her ideas and connections emerge, thereby facilitating the generation of new ideas and connections. The interviewee proceeds to relate concepts and make connections being prompted only as necessary by the interviewer with questions such as "what does this lead to?"

Analysis of the data involved entering handwritten concepts and links into a mapping software program (in this case Banxia Decision Explorer). Concepts were visually coded by using a different font and format for each of strategic goals, sub-goals, and action items. Concepts were arranged hierarchically and re-drawn for clarity to minimize the number of cross-links. The software was used to run a series of analyses on the data, exploring each concept for the number of direct connections, and total connections (the "centrality" of a concept). Each concept is scored based on the number of concepts and distance removed (number of links) of all other linked concepts, with diminishing scores the further removed. High scores for centrality therefore suggest "busy" concepts or those that are highly networked and central to the theme. Concept clusters that appear to stand apart from other sections of the map were also analyzed. A "loop" analysis was used to reveal reinforcing circular concepts.

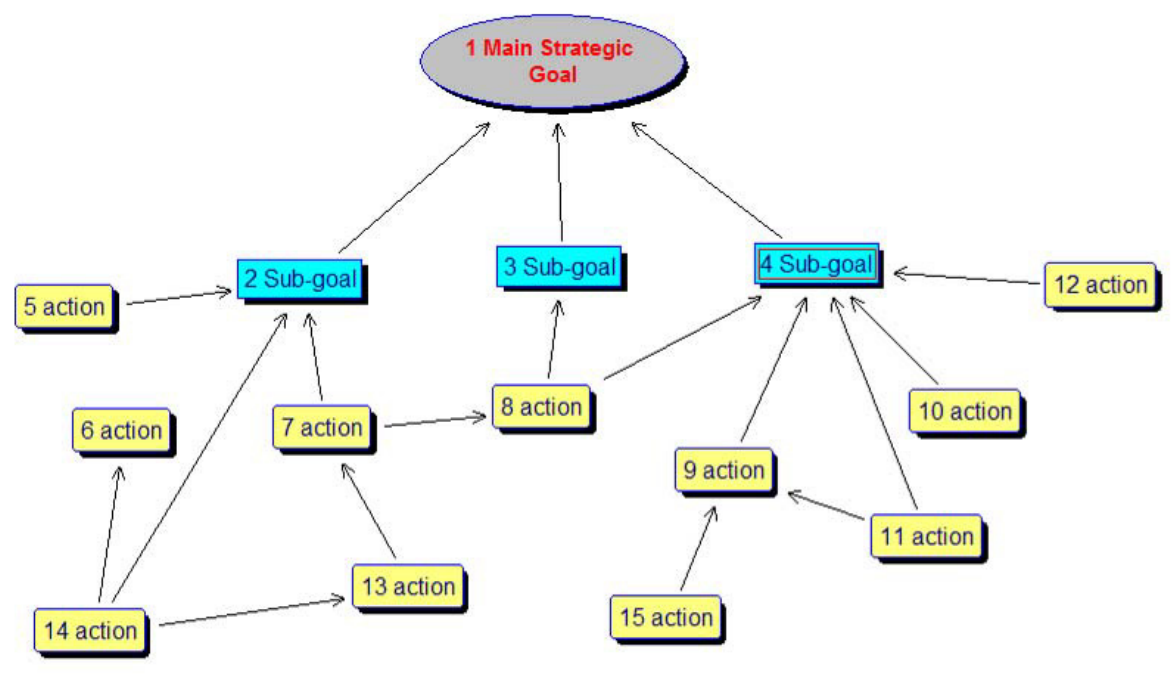

Fig. 1. Sample hierarchical cognitive map showing strategic goal at the top, sub-goals in the middle and numerous actions that led to goals. 


\section{Case Study Illustration of Cognitive Mapping}

The one-hour mapping session resulted in a total of 71 concepts. The interviewee suggested that the main strategic goal for many manufacturing companies is to release new products on a regular basis. This was supported by four sub-goals: increased product variety, rapid set-up, rapid operational ramp-up, and increased yield and throughput. The size and complexity of a highly networked map with 71 concepts makes it very difficult to read and comprehend. However, software analysis provides a number of alternate ways to parcel the data. The analysis of "central" concepts is revealing. In the sample map of the case study, the concepts most central to achieving strategic goals were: improve set-up of assembly; define chunks of work that can be run in parallel rather than serial; improve quality; improve work-rest ratios; and improve design for assembly to improve product design.

The software can then be used to draw smaller maps that focus on the central concepts to indicate action items that would support these. Figure 2 shows the action-related concepts that support "improving the design of the assembly to improve product design". Arrows leading away from the concept "improve design for assembly to improve product design" (box 14) may indicate that this would lead to not only the main strategic goal (release new products on a regular basis), but also improving error detection (box 16), correcting mis- takes quickly (box 15), improving assessments (box 72), and increasing flexibility (box 11). Arrows leading into the central concept indicate what actions achieve improving design of assembly. In Figure 2 , increased cognitive performance (of operators) (box 22) is believed to result in improved assembly design (box 14). Increased cognitive performance can be achieved by improving instructions for assembly (box 25) or by increasing control and reducing demands (on operators) (box 53). Continuing to disentangle the concepts shows four action items that link into improving instructions for assembly. This concept also leads to reduced need to learn (assembly becomes more automatic) (box 24). Figure 2 also shows that improving psychosocial factors (box 51) via increased control and reduced demands leads to increased cognitive performance. A number of other concepts also lead to improving psychosocial factors. Review of the smaller maps more clearly illustrates the link between actions typically considered in the realm of human factors (such as improve positioning of tools, improve psychosocial factors) and production outcomes (such as fewer errors), and main strategic goals (release new product on a regular basis). These concepts may not have been directly apparent even to the interviewee until they are drawn, visualized and linked.

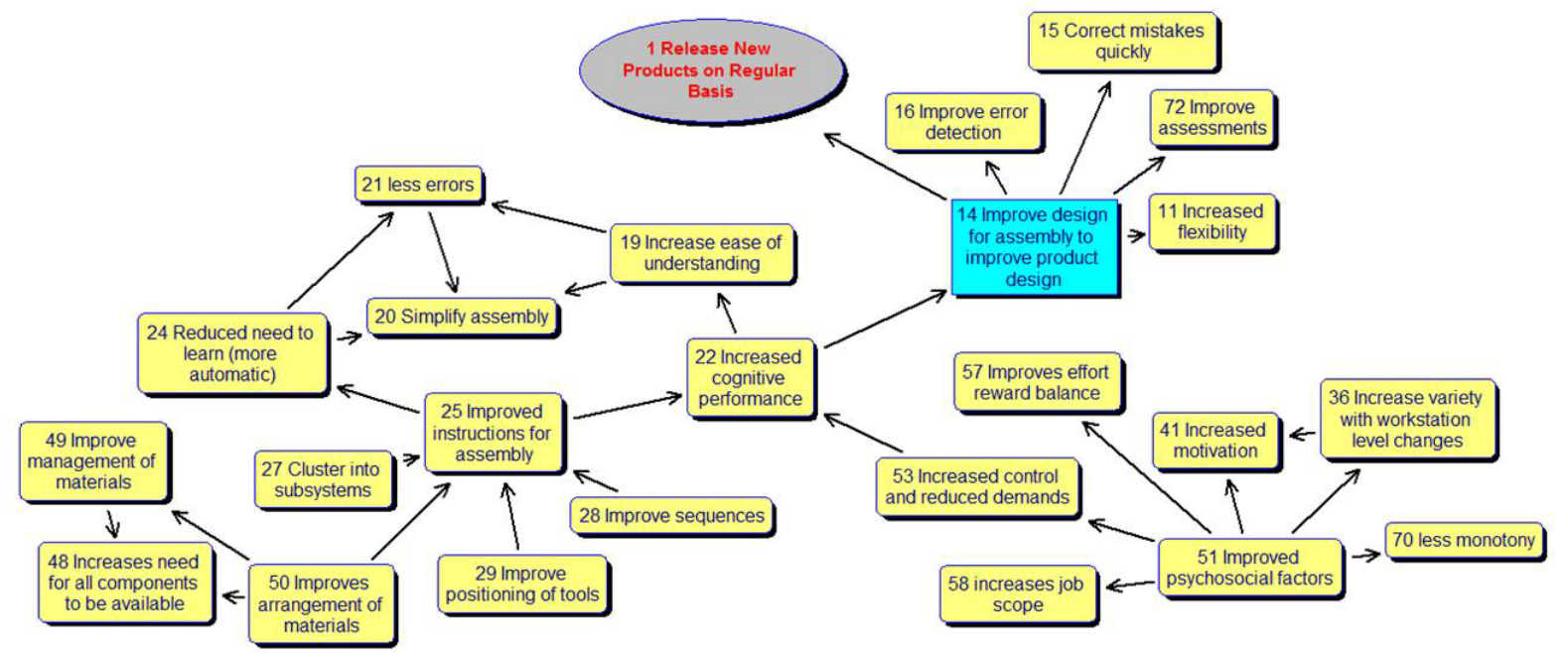

Fig. 2. Section of the cognitive map with one strategic goal (circle), one sub-goal (blue square) and immediate links 
The analysis also revealed 37 loops in the concept map. Loops are especially interesting as they show examples of actions that reinforce other actions in either positive or negative ways (damping). Figure 3 shows a smaller map with loops showing various ways to reduce worker pain and fatigue. In this figure, for example, "improved psychosocial factors" leads to "increased variety with workstation level changes", which in turn leads to "reduced worker pain and fatigue". The loop continues as "reduced worker pain and fatigue" leads to "improved psychosocial factors". Therefore, improvement in any one of these three factors will lead to subsequent impacts on the other factors, forming a possible 'virtuous' feedback loop.

\section{Discussion and Applications}

A cognitive map can be described as an exploratory route map of a person's knowledge and understanding of a topic at a given time [8]. Similar techniques have been used for many years in the educational sciences to explore students' understanding in science, engineering, nursing, teaching, business, enterprise and the arts. Hay and colleagues [7] suggest that mapping students' prior knowledge gives teachers an idea of where to start with planning learning content. It also reveals any misconceptions in the map. There is some suggestion that the higher the number of concepts and more linkages, the more "expert" the knowledge of the participant in a given topic. Therefore a comparison of maps at two points in time can reveal "learning".
Similarly cognitive mapping, therefore, could be a useful technique to explore the knowledge and understanding of human factors among senior managers, and likewise reveal any misconceptions or gaps. Mapping the HF knowledge of different managers may provide a useful benchmark for planning $\mathrm{HF}$ initiatives or programs. There is also potential to perform the mapping exercise pre-and-post HF initiatives or programs to evaluate "learning" on the part of senior managers.

The one-hour interview conducted in this case study resulted in 71 concepts pertaining to HFs. This is consistent with reports in the literature suggesting 30-120 concepts can be mapped from a one-hour interview [6]. As a method of condensing qualitative data, the mapping exercise can be a very timeeffective way for HF practitioners to learn about the organizational priorities and strategic goals in a company and to compare these perceptions between different managers.

Outside of the educational field, cognitive maps have been used in business and organizations and in qualitative research to effectively reduce qualitative data to a more manageable level, to analyze themes and interconnections, and to present findings [3]. This single case study confirmed the experience reported by others that the mapping process stimulates deeper responses from participants compared with traditional interviews, and allows them to correct misunderstandings and make links that may otherwise not be apparent. The visual nature of mapping allows for evidence checking and can more

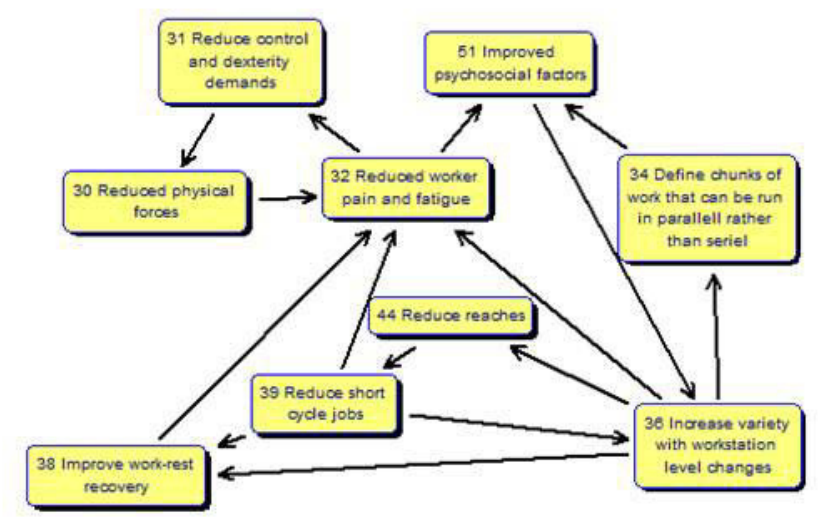

Fig. 3. Part of a cognitive map showing one of the many reinforcing loops between concepts 
fully lead to saturation on a topic than other methods. The visual nature may also lead to enhancing or expanding the conceptual framework by raising other questions and opening up the enquiry. It has been suggested that emergent themes can arise [3]. It is anticipated that cognitive mapping could help senior managers make the connections between HF and their strategic goals that otherwise may not have been immediately apparent to them. This should theoretically lead to increased management support for $\mathrm{HF}$ in the organization.

Cognitive and concept mapping are also being recognized in the management literature, both in strategic management and in operational research [11]. Mapping has been used with individuals and with groups to investigate business decline, rationale for joint ventures and management perceptions of competitive positioning, as well as in group model building for dynamic decision-making [11]. Mapping has also been used to measure team mental models to facilitate teamwork for a task [12]. Lee et al [10] suggests that organizational learning can be facilitated by group map making. As group members share their concept maps, they see their concepts in relation to each others, thereby producing learning. If a "group" map were created by senior managers linking HF to strategic goals, it is anticipated that the map-making process would facilitate their learning about HFs from one another.

Finally, cognitive mapping appears to be useful for solving problems and indicating alternative actions [13]. The map produced in this case study showed numerous opportunities for increasing the application of HF that by various routes would also result in improved performance and help an organization achieve its strategic goals.

We are currently exploring the use of this technique with key stakeholders to learn about the organizations' strategic goals, enhance the role of HF within the organization, and identify initiatives that support the role of HF. An industry based case study trial is in progress to further examine the utility of this tool to help a managerial team capture the strategic benefits of applying HF in their operations.

\section{Conclusions}

Cognitive mapping, a technique that has been successfully used in management and operational research literature, may be useful to human factors practitioners. It can be used with senior managers to explore the link between human factors and strategic goals of the organization or with a group of managers to determine the most effective actions to support HF in line with strategic goals. The sample trial reported here showed numerous human factors actions that would support strategic goals. An industry trial is underway to further evaluate this technique.

\section{References}

[1] F. Ackermann, C. Eden and S. Cropper, Getting started with cognitive mapping, (13-15 $\left.{ }^{\text {th }}\right)$ April 1992, University of Warwick, 65-82.

[2] S. M. Brown, Cognitive Mapping and Repertory Grids for Qualitative Survey Research: Some Comparative Observations. Journal of Management Studies 29(3) (1992) 287-307.

[3] B. J. Daley,Using concept maps in qualitative research. In: Canas AJ, Novak, J.D., Gonzalez, F.M., editor; 2004; Pamplonia, Spain. pp7.

[4] C. G. Drury, Human factors and quality: Integration and new directions. Human Factors and Ergonomics in Manufacturing 10(1) (2000) 45-59.

[5] J. Dul, and P. Neumann, Ergonomics contributions to company strategies. Applied Ergonomics 40 (2009) 745-752.

[6] C. Eden, Analyzing cognitive maps to help structure issues or problems. European Journal of Operational Research 1(59) (2004) 673-686.

[7] D. Hay, I. Kinchin, and S. Lygo-Baker, Making learning visible: the role of concept mapping in higher education. Studies in Higher Education 33(2) (2008) 295-311.

[8] D. B. Hay and I. M. Kinchin, Using concept maps to reveal conceptual typologies. Education + Training 48(2/3) (2006) 127-142.

[9] S. Howick, C. Eden, F. Ackermann, and T. Williams, Building confidence in models for multiple audiences: The modelling cascade. European Journal of Operational Research, 186 (2008) 1068-1083.

[10] S. Lee, J. F. Courtney, and R. M. O'Keefe Jr., A System for Organizational Learning Using Cognitive Maps. Journal of Management Science 20(1) (1992) 23-36.

[11]J. D. W. Moreroft, Executive knowledge, models and learning. European Journal of Operational Research 59 (1992) 927.

[12]D. L. O'Connor, T. E. Johnson and M. K. Khalil, Measuring team cognition: Concept mapping elicitation as a means of constructing team shared mental models in an applied setting. In: Canas AJ, Novak, J.D., Gonzalez, F.M., editor; 2004; Pamplona, Spain. p 1-7.

[13]J. Swan, Using cognitive mapping in management research: Decisions about technical innovation. British Journal of Management 8 (1997) 183-198.

[14]D. P. Tegarden and S. D. Sheetz, Group cognitive mapping: a methodology and system for capturing and evaluating managerial and organizational cognition. Omega, 31 (2003) 113-125. 